\title{
Possible New Strange Attractors With Spiral Structure
}

\author{
A. Arneodo ${ }^{1}$, P. Coullet ${ }^{2}$, and C. Tresser ${ }^{2}$ \\ 1 Laboratoire de Physique Théorique, Université de Nice, Parc Valrose, F-06034 Nice Cedex, France* \\ 2 Equipe de Mécanique Statistique, Université de Nice, Parc Valrose, F-06034 Nice Cedex, France ${ }^{\star \star}$
}

\begin{abstract}
We define a class of three-dimensional differential equations which might present strange attractors of a new kind. This is illustrated by numerical observations on an explicit example.
\end{abstract}

\section{Introduction}

These last years a lot of efforts have been devoted to the study of chaotic behaviours which may arise in some dynamical systems. The relevance of these works to understand the transition to turbulence in physical and chemical experiments seems now well established [1]. A mathematical theory of Axiom A "strange attractors" has been fairly achieved and provides a satisfying description of some systems which do have complicated asymptotic behaviour $[2,3]$. Nevertheless this theory does not allow us to understand the stochasticity generated in numerical investigations of diffeomorphisms and differential equations given by explicit algebraic expressions. Indeed most of these numerical studies deal with generalizations of the Henon mapping $[4,5]$ or its suspensions [6]. The existence of chaotic behaviour can be proved in these cases [7-10] but, according to [11], the very existence of strange attractors is far from being settled since homoclinic tangencies [12] arise quite naturally with one-parameter families of such systems ${ }^{1}$. Perhaps a special status should be attributed to the Lorenz system [14] which could fall in the class of topological models defined by Guckenheimer [15] and which posses effective strange attractors [16,17].

In this paper we propose a construction of what might reveal to be a new kind of strange attractors. This construction is illustrated by numerical observations on an explicit example of differential equations describing a forced oscillator [18].

* Equipe de Recherche Associée au C.N.R.S.

$\star \star$ Laboratoire de Physique de la Matière Condensiée Associé au C.N.R.S.

1 Recently Misiurewicz [13] proved the existence of strange attractors for the homeomorphism $(x, y) \rightarrow(1-a|x|+b y, x)$, under some conditions on the parameters $a$ and $b$ 


\section{A Class of Differential Equations}

Our main tool is the following theorem:

Theorem. Consider the system

$$
\left\{\begin{array}{l}
\dot{x}=\varrho x-\omega y+P(x, y, z) \\
\dot{y}=\omega x+\varrho y+Q(x, y, z) \\
\dot{z}=\lambda z+R(x, y, z)
\end{array}\right.
$$

where $P, Q, R$ are $C^{r}$ functions $(1 \leqq r \leqq \infty)$ vanishing together with their first derivatives at the origin $0=(0,0,0)^{2}$. Let us assume that one of the orbits, denoted by $\Gamma_{0}$, leaves the origin and returns to it as $t \rightarrow+\infty$. Then if

$$
\lambda>-\varrho>0
$$

every neighbourhood of the orbit $\Gamma_{0}$ contains a denumerable set of unstable periodic solutions of saddle type.

Proof. This theorem was proved in [19] under the inessential assumption that $P$, $Q, R$ are analytic functions in $\mathbb{R}^{3}$. The associated symbolic dynamics was investigated in any dimension by the same author in [20] where it is shown that a Birkhoff-Morse system (shift with infinitely many symbols) is contained in a neighbourhood of the homoclinic trajectory $\Gamma_{0}$.

We will however sketch a proof of the above theorem based on a geometrical construction (Fig. 1) which explains the structure of the attractor we shall get.

Following Hartman-Grobman theorem $[21,22]^{3}$, instead of (1) we can work with a vector field which is conjugate to (1) and linear in some neighbourhood $V$ of the origin 0 (then the local invariant manifolds of 0 are linear in $V$ ).

Let $\Omega_{0}$ be a point of the homoclinic curve $\Gamma_{0}$ close enough to the saddle focus 0 , and $\Pi_{0}$ part of a plane orthogonal to the local stable manifold $W_{\text {loc }}^{s}$ of 0 and containing $\Omega_{0}$. We define $P_{0}$ as the Poincaré's first return map on $\Pi_{0}$. Then let $A_{1} \in \Pi_{0}$ and $A_{1}^{\prime}=P_{0}\left(A_{1}\right)$. We can construct $B_{1}^{\prime}$ and $B_{1}$ in $\Pi_{0}$ such that $A_{1} B_{1}^{\prime} A_{1}^{\prime} B_{1}$ is a rectangle (we choose $A_{1}$ such that the distances from $\Omega_{0}$ to $A_{1}$ and $B_{1}^{\prime}$ are equal). We set $A_{2}^{\prime}=B_{1}^{\prime}$ and $A_{2}=P_{0}^{-1}\left(A_{2}^{\prime}\right)$. Then continuing this iterative procedure, we generate a sequence of rectangles $\left\{A_{i} B_{i}^{\prime} A_{i}^{\prime} B_{i}\right\}$ in $\Pi_{0}$.

Let $\Omega_{1}$ be a point of the local unstable manifold $W_{\text {loc }}^{u}$ of 0 , close enough to 0 , and $\Pi_{1}$ part of a plane orthogonal to $W_{\text {loc }}^{u}$ and containing $\Omega_{1}$. We define $T_{0}: \Pi_{0} \rightarrow \Pi_{1}$ as the map which associates to each point $M$ of $\Pi_{0}$, the first intersection with $\Pi_{1}$ of the orbit issued from $M$. Then the family of rectangles $\left\{A_{i}\right.$ $\left.B_{i}^{\prime} A_{i}^{\prime} B_{i}\right\}$ transforms under $T_{0}$ as represented on Fig. 1.

Now let $T_{1}: \Pi_{1} \rightarrow \Pi_{0}$ be the map which associates to each point $M$ of $\Pi_{1}$, the first intersection with $\Pi_{0}$ of the orbit originating from $M$. Then $T=T_{1} \circ T_{0}$ maps $\Pi_{0}$ into itself. The above theorem results from the fact that if $A_{1}$ is close enough to $W_{\text {loc }}^{s}$ then (2) and the regularity of the vector field imply that for each $i,\left.T\right|_{A_{i} B_{i}^{i} A_{i}^{\prime} B_{t}}$ is a topological horseshoe $[2,7]$.

2 Using Poincaré's terminology, the origin is then a saddle focus

3 Actually a $C^{r}$ linearization $(1 \leqq r \leqq \infty)$ would be available under mild (non resonance) assumptions on eigenvalues [23]. This let $T_{1}$ regular enough. One then uses density of non resonant systems and stability of topological horseshoes to conclude 


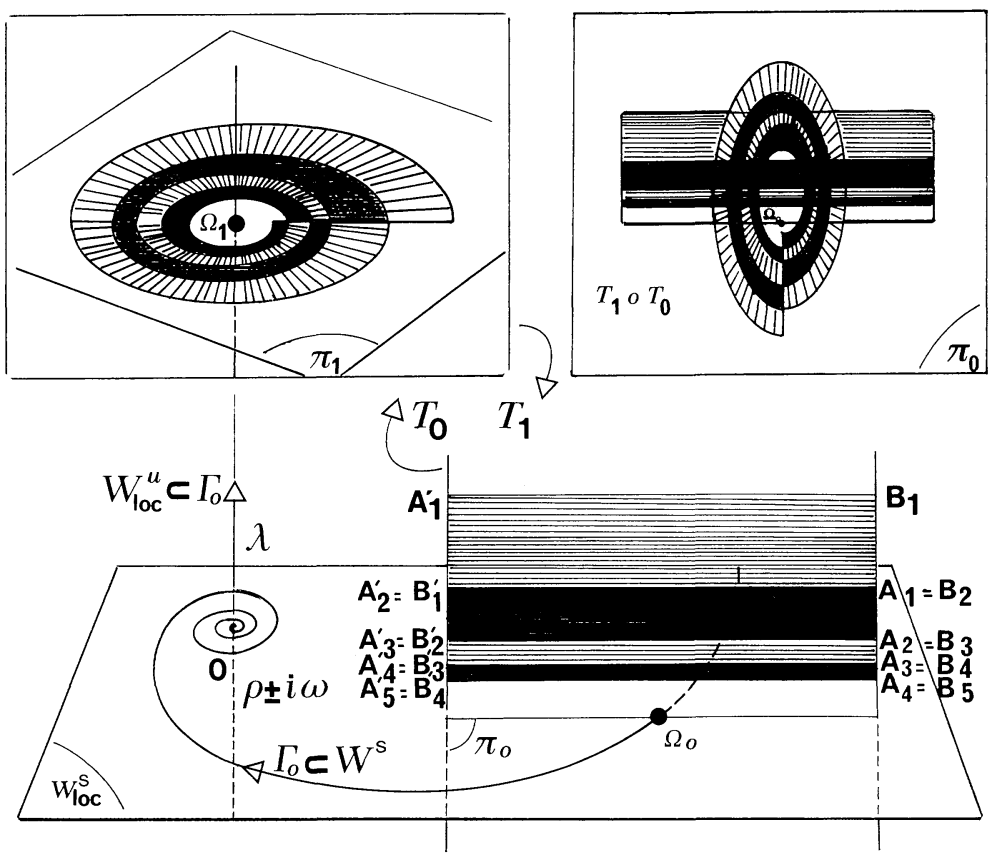

Fig. 1. Geometrical construction used for the proof of Shil'nikov's theorem. We have also represented how acts the map $T=T_{1} \circ T_{0}$

At this point let us remark that $T$ maps parts of each rectangle $A_{i} B_{i}^{\prime} A_{i}^{\prime} B_{i}$ on the opposite side of $W_{\mathrm{loc}}^{\mathrm{s}}$, which prevents defining a $T$-invariant region in $\Pi_{0}$. This leads us to specify the dynamics on both side of $W_{\text {loc }}^{s}$. We proceed in a simple way by imposing on (1) the symmetry with respect to the origin: $(x, y, z) \rightarrow(-x,-y,-z)$. Then if $\bar{A}_{1}^{\prime}$ and $\bar{B}_{1}$ are symmetric to $A_{1}^{\prime}$ and $B_{1}$ with respect to $W_{\mathrm{loc}}^{s}\left(W_{\mathrm{loc}}^{s}\right.$ is linear in $\left.V\right)$, we can find systems such that, as soon as $A_{1}$ is far enough from $W_{\mathrm{loc}}^{s}$, the set theoretical complementary of $W_{\mathrm{loc}}^{s}$ in the open rectangle $\bar{B}_{1} \bar{A}_{1}^{\prime} A_{1}^{\prime} B_{1}$, is invariant under the Poincare first-return map defined on it. (The rectangles $\left\{A_{i} B_{i}^{\prime} A_{i}^{\prime} B_{i}\right\}$ with $i$ large enough still support topological horseshoes.)

For such systems it is obvious that $T_{0}\left(\bigcup_{i=1} A_{i} B_{i}^{\prime} A_{i}^{\prime} B_{i}\right)$ is invariant under the Poincaré first-return map $P_{1}$ defined on $\Pi_{1}$. Using the foliation depicted in Fig. 1, we can conclude that any attractor of $P_{1}$ must be included in a spiral asymptotic to $\Omega_{1}$.

\section{An Example}

Now we come to the numerical investigation of a specific example choosen in a class of differential equations introduced in [18].

$$
\left\{\begin{array} { l } 
{ \ddot { x } + \beta \dot { x } + x = \eta } \\
{ \dot { \eta } = f _ { \mu } ( x ) }
\end{array} \text { or } \left\{\begin{array}{l}
\dot{x}=y \\
y=z \\
\dot{z}=-y-\beta z+f_{\mu}(x),
\end{array}\right.\right.
$$




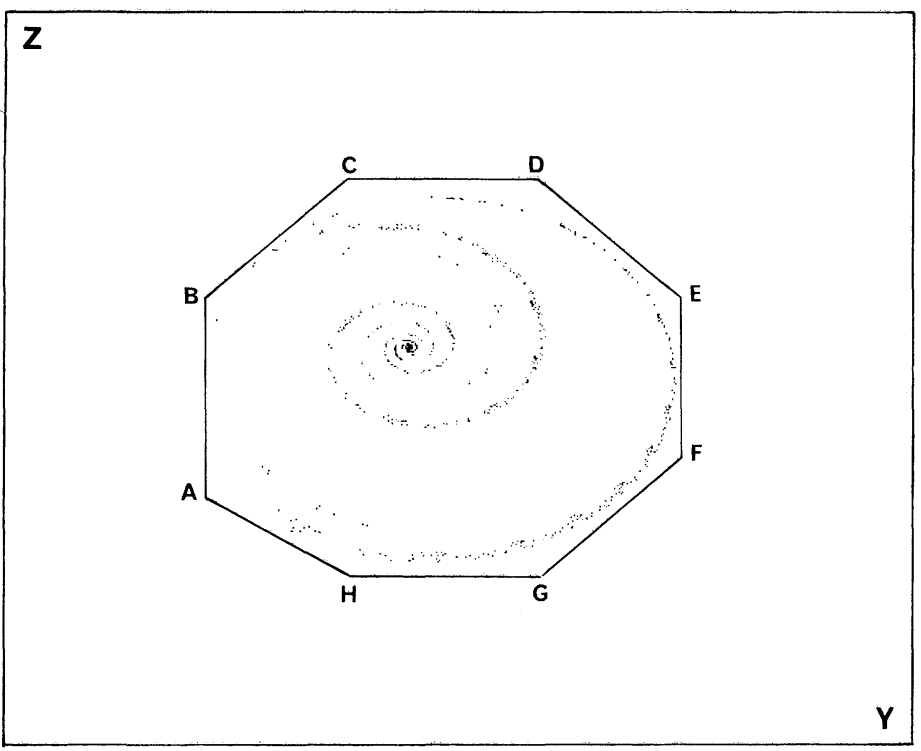

Fig. 2. For system (3) with $f_{\mu}(x)$ given by (5) and $a \simeq 0.2171604$ and $\mu \simeq 0.2061612$, the image under $P_{1}$ of a lattice of 1000 points taken in the polygon $A B C D E F G H$ with $A(0.000001,-0.162), B(0.000001$, $0.108), C(0.141,0.270), D(0.329,0.270), E(0.470,0.108), F(0.470,-0.108), G(0.329,-0.270), H(0.141$, $-0.270)$

where $\beta>0$ is the dissipation and $f_{\mu}$ a real function depending on the parameter $\mu$. In a previous paper [24], we considered system (3) with the particular choice

$$
f_{\mu}(x)=\left\{\begin{array}{lll}
1+a x & \text { if } & x \leqq 0 \\
1-\mu x & \text { if } & x \geqq 0
\end{array}\right.
$$

and proved the existence for some $a_{0}, \mu_{0}$ of a homoclinic orbit $\Gamma_{0}$ satisfying the conditions of Shil'nikov's theorem [19]. This allowed us to understand the structure of the chaotic behaviours numerically displayed by (3) for values of $\mu$ less than $\mu_{0}$. Nevertheless let us emphasize that for such functions $f_{\mu}(x)$ with only one extremum, no attractor is observable simultaneously with $\Gamma_{0}$ [24]. In order to remedy this fact, we impose the above mentioned symmetry $(x, y, z) \rightarrow(-x,-y$, $-z$ ) by choosing $f_{\mu}(x)$ as defined by

$$
f_{\mu}(x)=\left\{\begin{array}{ccc}
-\mu x-\mu-a & \text { if } & x \leqq-1 \\
a x & \text { if } & |x| \leqq 1 \\
-\mu x+\mu+a & \text { if } & x \geqq 1
\end{array} .\right.
$$

Two main reasons lead us to consider a piecewise linear function ${ }^{4,5}$ :

i) Using the method developed in [24], we can prove the existence of values of the parameters such that there exist two homoclinic orbits $\Gamma_{0}$ and $\Gamma_{0}^{\prime}$ symmetric with respect to the saddle focus $(0,0,0)$, all conditions of Shil'nikov's theorem being fulfilled.

4 Analogous results have also been obtained with analytical functions

5 The above theorem is readily adapted to this case 


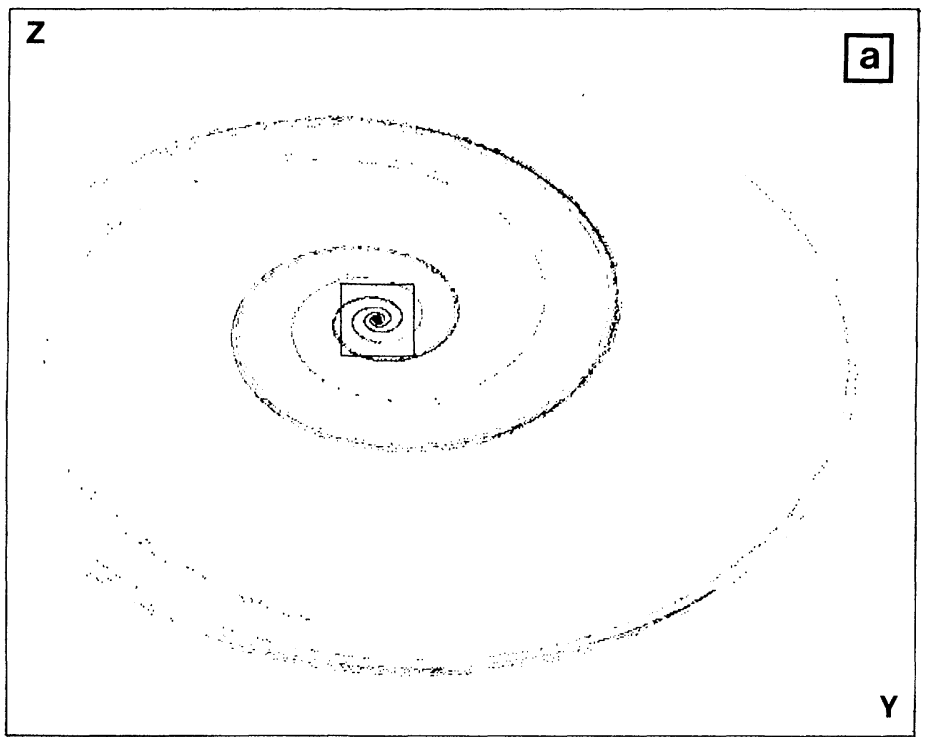

$\mathbf{Z}$

Fig. 3. a The spiral like attractor numerically observed for $P_{1} ; \mathbf{b}$ Magnification of the boxed-in region in a same model as in Fig. 2

ii) The solutions of (3) can be computed with a very accurate numerical scheme: the solutions are known analytically in each of the strata $x \leqq-1,|x| \leqq 1$ and $x \geqq 1$, and we only need to compute with Newton's method the return times on the planes

$$
x=-1 \text { and } x=+1 .
$$

Our numerical procedure makes easy the computation of the Poincare first return map on the plane $x=1$, which we consider as including $\Pi_{1}$. Figure 2 gives 


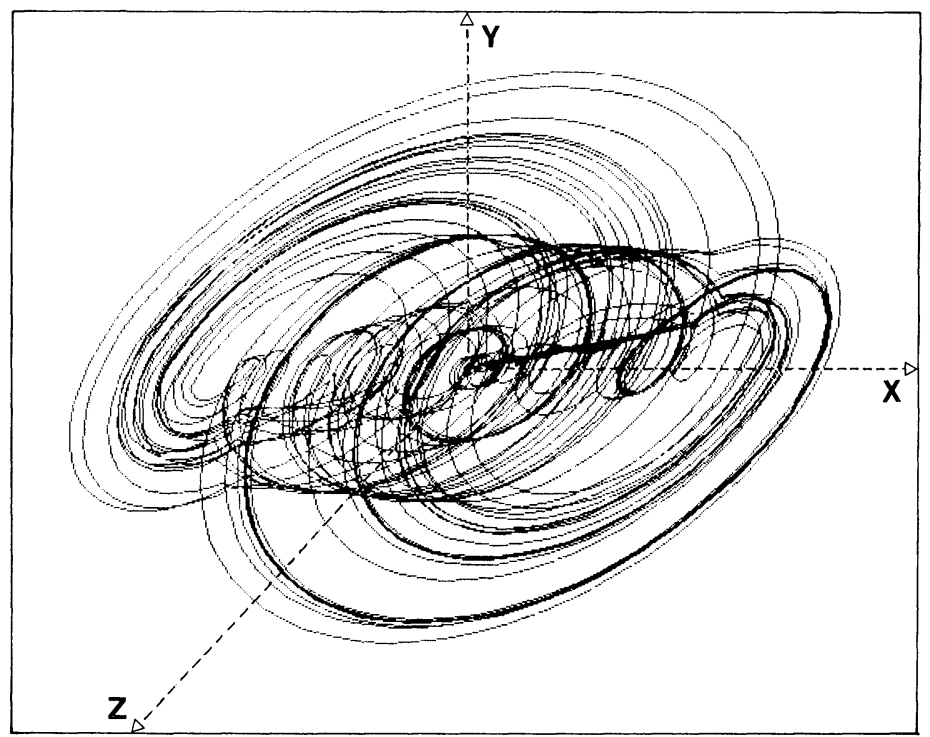

Fig. 4. A three-dimensional representation of the attractor; same model as in Fig. 2

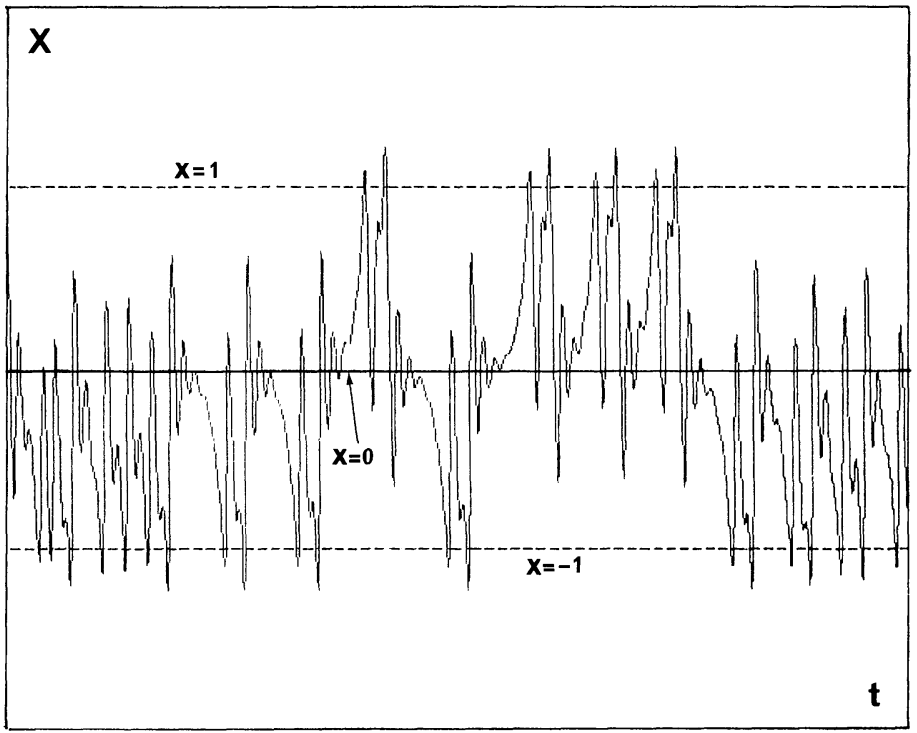

Fig. 5. A typical sample of the time evolution of the $x$-coordinate; same model as in Fig. 2

evidence that this Poincaré map $P_{1}$ admits an invariant polygonal surface for values of the parameters such that $\Gamma_{0}$ and $\Gamma_{0}^{\prime}$ exist $\left(a_{0} \simeq 0.2171604\right.$, $\mu_{0} \simeq 0.2061612$ ). The corresponding attractor is represented in Fig. 3a, where the spiral structure previously predicted is clearly observed (this spiral structure can be guessed on a 3-dimensional plot of the attractor of the corresponding flow: See Figs. 4 and 5). The magnification of the boxed-in region (Fig. 3b) strongly suggests 
that the attractor is scale-invariant and comes in each neighbourhood of $\Omega_{1}$ which has no image under $P_{1}$. If it turns out to be true, the attractor cannot be a periodic orbit, and we conjecture that we are faced with a strange attractor of a new kind.

\section{Conclusion}

We must emphasize that when we increase the parameter $\mu$ from 0 to the value $\mu_{0}$ where $\Gamma_{0}$ and $\Gamma_{0}^{\prime}$ exist, the dynamics associated with system (3) becomes more and more complicated. The initial evolution (when observed on an arbitrary Poincaré first-return map) is similar to what is obtained when strengthening the nonlinearities in the Henon mapping [4]. Further evolution corresponds to a succession of topological horseshoes with more and more branches i.e. semiconjugate to full shifts on a alphabet with more and more symbols.

Acknowledgements. We would like to thank D. Ruelle who brought to our knowledge that Ya. Sinai already envisaged the possible existence of attractors with spiral structure. We are also grateful to J. Palis and R. F. Williams for encouraging comments, and to A. Chenciner for a careful reading of a preliminary version of the manuscript.

\section{References}

1. Gurel, O., Rössler, O.E.: Ann. N.Y. Acad. Sci. 316 (1979)

2. Smale, S.: Bull. Am. Math. Soc. 73, 747 (1967)

3. Bowen, R., Ruelle, D.: Invent. math. 29, 181 (1975)

4. Henon, M.: Commun. Math. Phys. 50, 69 (1976)

5. Curry, J.H., Yorke, J.A.: The structure of Attractors in dyn. systems. Markley et al. (ed). In: Lecture notes in mathematics. Vol. 668, 48, Berlin, Heidelberg, New York: Springer 1978

6. Rössler, O.E.: Phys. Lett. 57 A, 397 (1976)

7. Devaney, R., Nitecki, Z. : Commun. Math. Phys. 67, 137 (1979)

8. Marotto, F.R. : Commun. Math. Phys. 68, 187 (1979)

9. Misiurewicz, M., Szewc, B.: Warszawa Preprint (1979)

10. Tresser, C., Coullet, P., Arneodo, A.: J. Phys. A 13, L-123 (1980)

11. Newhouse, S.: Publ. I.H.E.S. 50, 101 (1979)

12. Newhouse, S., Palis, J.: Dynamical systems. Peixoto, M. (ed.). New York: Academic Press 1973

13. Misiurewicz, M.: Talk given at the International Conference on Nonlinear Dynamics held at NewYork on December 1979

14. Lorenz, E.N.: J. Atmos. Sci. 20, 130 (1963)

15. Guckenheimer, J.: Hopf bifurcation and its applications. Marsden, J.E., Mc Craken, M. (eds.). In : Applied Mathematical Science, Vol. 19. New York: Springer 1976

16. Guckenheimer, J., Williams, R.F.: Publ. I.H.E.S. 50, 59 (1979)

17. Williams, R.F.: Publ. I.H.E.S. 50, 73 (1979)

18. Coullet, P., Tresser, C., Arneodo, A. : Phys. Lett. 72 A, 268 (1979)

19. Shil'nikov, L.P.: Sov. Mat. Dok. 6, 163 (1965)

20. Shil'nikov, L.P.: Mat. USSR. Sb. 10, 91 (1970)

21. Hartman, P.: Ordinary differential equations. New-York: Wiley 1964

22. Grobman, D.: Dokl. Akad. Nauk. USSR 128, 880 (1965)

23. Sternberg, S.: Am. J. Math. 80, 623 (1958)

24. Arneodo, A., Coullet, P., Tresser, C.: Nice Preprint NTH 80/7

Communicated by D. Ruelle

Received July 10, 1980; in revised form October 1, 1980 
